\title{
MONITORAMENTO DA TERAPIA ANTIRRETROVIRAL PARA O HIV EM UMA UNIDADE DISPENSADORA DE MEDICAMENTOS
}

\section{MONITORING OF THE ANTIRETROVIRAL THERAPY FOR HIV IN A DISPENSING UNIT OF MEDICATIONS.}

\section{RESUMO}

OBJETIVOS: Verificar o perfil e adesão ao tratamento de pessoas vivendo com HIV ou aids que iniciaram a Terapia antirretroviral (TARV) no ano de 2015, em Unidade Dispensadora de Medicamentos antirretrovirais de um hospital Público. MÉTODOS: Os dados foram obtidos através de busca nos registros do Sistema de Controle Logístico de Medicamentos Antirretrovirais. RESULTADOS: houve predominância do sexo masculino, sendo o grau de escolaridade de 58,4\% dos pacientes maior que oito anos. Gestantes apresentaram alta adesão a TARV. A infecção por transmissão vertical foi detectada em $1,05 \%$ dos casos. Houve $11,83 \%$ de abandono de tratamento, sendo possível acompanhar por pelo menos seis meses 156 pacientes. Entre estes 88,5\% apresentaram alta adesão a TARV, 3,84\% adesão inadequada e 7,7\% adesão insuficiente. CONCLUSÕES: O perfil mostrou-se semelhante ao encontrado em outras regiões do Brasil. A adesão a TARV mostrou-se satisfatória especialmente entre gestantes, refletindo na baixa frequência de crianças infectadas verticalmente.

Palavras-Chave: HIV, Tratamento Medicamentoso, Medicamentos de Dispensação Assistida.

\section{ABSTRACT \\ OBJECTIVES: To verify the profile and adherence to the treatment of people living with HIV or AIDS who started antiretroviral therapy (ART) in 2015, in an Antiretroviral Drug Dispensing Unit of a Public hospital. METHODS: The data were obtained by searching the records of the Logistic Control System of Antiretroviral Medications. RESULTS: there was a predominance of males, and the educational level of $58.4 \%$ of the patients was greater than eight years. Pregnant women showed high adherence to ART. Vertical transmission infection was detected in $1.05 \%$. There was $11.83 \%$ of treatment abandonment, being possible to follow up for at least six months 156 patients. Among these, $88.5 \%$ had high adhesion to HAART, 3.84\% inadequate adhesion and $7.7 \%$ insufficient adhesion. CONCLUSIONS: The profile was similar to that found in other regions of Brazil. Adherence to ART was satisfactory especially among pregnant women, reflecting the low frequency of vertically infected children.}

KeyWords: HIV, Drug Therapy; Behind-the-Counter Drugs

Sandra Trevisan Beck Correio
http://lattes.cnpq.br/4435727183593265
Universidade Federal de Santa Maria
Brasil
Curso de Farmacia, departamento de análises
clínicas e toxicológicas.
Marina Zankoski Correio
http://lattes.cnpq.br/9097132152065336
Universidade Federal de Santa Maria
Brasil
Academica do Curso de Farmácia
Sarmaceutica, mestre, Hospital Universitário de
Claudia Sala Andrade Correio
http://lattes.cnpq.br/2863361741058060
Universidade Federal de Santa Maria
Santa Maria
Laura Vielmo Correio
http://lattes.cnpq.br/1524946907055427
Universidade Federal de Santa Maria
Brasil

http://lattes.cnpq.br/4435727183593265

Brasil

Curso de Farmacia, departamento de análises
clínicas e toxicológicas.

Marina Zankoski Correio

http://lattes.cnpq.br/9097132152065336

Universidade Federal de Santa Maria

Brasil

Laura Vielmo Correio

http://lattes.cnpq.br/1524946907055427

Universidade Federal de Santa Maria

Brasi

Farmaceutica, mestre, Hospital Universitário de Santa Maria 


\section{Introdução}

O Vírus da Imunodeficiência Humana (HIV) é um retrovírus que ataca e destrói as células do sistema imunológico, principalmente os Linfócitos T CD4+ (LT CD4+) que são cruciais para uma resposta imune satisfatória. As manifestações clínicas e complicações decorrentes desta infecção pelo HIV é o que desencadeia a Síndrome da Imunodeficiência Adquirida (aids), sendo importante o diagnóstico precoce.1 Conforme atualização da Portaria - 204, de 17 de fevereiro de 2016, a infecção pelo HIV e a AIDS fazem parte da Lista Nacional de Notificação Compulsória de doenças, sendo a notificação compulsória da infecção pelo HIV muito recente2.

Paradetecção e atendimento destes pacientes vivendo com aids e/ou HIV, os Centros de Testagem eAconselhamento (CTA), Serviços de Assistência Especializada e Unidades Dispensadoras de Medicamentos (UDM) com incorporação gradual de Unidades Básicas de Saúde vem prestando atendimento de forma cada vez mais aprimorada.3 Isto é de extrema relevância uma vez que proporcionando o diagnóstico precoce da infeç̧ão pelo HIV é possível aumentar a expectativa de vida do paciente, que ao fazer o diagnóstico terá acesso ao tratamento adequado no tempo correto1. 0 objetivo da Terapia Antirretroviral (TARV) é a diminuição da Carga Viral (CV) e aumento dos níveis de LT CD4+ uma vez que se observa que pessoas em uso de TARV, que mantêm contagens de LT CD4+ acima de 500 células $/ \mathrm{mm} 3$ e CV indetectável ou abaixo do limite de detecção ( 50 cópias virais/ $\mathrm{mL}$ de sangue), atingem expectativa de vida semelhante à da população geral.4

No Brasil, nos dias de hoje, 489.000 mil pessoas têm acesso gratuitamente a TARV2 devido à política de distribuição universal e gratuita dos medicamentos antirretrovirais para as pessoas vivendo com HIV ou AIDS, que teve inicio em 1996 através da Lei 9.313 que declara que os portadores do HIV e indivíduos com a aids terão direito a receber gratuitamente do Sistema Único de Saúde, toda a medicação necessária a seu tratamento. Grande parte desses medicamentos ê produzida em laboratórios nacionais, sendo esta uma das estratégias para combater a doença5. Os efeitos positivos dessa política envolvem além da redução da mortalidade, diminuição das internações hospitalares, redução da incidência de infecções oportunistas, a prevenção da transmissão vertical onde a gestante infectada não tratada pode transmitir o HIV ao recém-nascido no período intra-utero, no momento do parto ou através do aleitamento materno6.

Mais recentemente, tem-se observado que o início mais precoce de TARV reduz também a transmissão do HIV, devido indivíduos com carga viral indetectável não transmitirem o vírus através de relação sexual. Desta forma, recomenda-se estimular início imediato da TARV para todas as pessoas vivendo com HIV ou aids, independentemente da contagem de LT CD4+na perspectiva de redução da transmissibilidade do HIV7.

Ressalta-se que uma vez iniciada a TARV, esta não pode ser interrompida. Desta forma, visando uma melhor eficácia do tratamento por longo tempo, tem havido uma tendência mundial buscando simplificar os esquemas de tratamento. A apresentação da formulação da dose fixa, combinada em uma só pílula (3 em 1) dos antirretrovirais Tenofovir, Lamivudina e Efavirenz , ao diminuir o número de comprimidos a serem ingeridos, veio facilitar a adesão do paciente ao tratamento uma vez que a terapia está baseada em apenas uma administração diária8. Com isto, é possível alcançar a efetividade terapêutica desejada, uma vez que é necessária uma adesão rigorosa aos medicamentos prescritos para ser obtida uma supressão viral sustentada, sendo considerado o ideal a ingestão igual ou superior a $95 \%$ das doses prescritas 9 . Outro benefício da ingestão correta da medicação é evitar o aparecimento de cepas resistentes aos antirretrovirais, uma vez que uma baixa adesão pode representar uma ameaça à saúde pública, pelo aumento de probabilidade de surgimento destas cepas resistentes, proporcionando um tratamento com baixa perspectiva de controle da replicação do HIV, bem como na disseminação de um vírus multirresistente 10.

Sabe-se que o inicio do tratamento é definidor do perfil de adesão do usuário. Isto se deve ao fato de às manifestações do HIV serem frequentemente assintomáticas no decorrer da infecção e com o inicio da TARV poderem surgir efeitos colaterais decorrentes dos medicamentos ingeridos, o que acaba prejudicando a adesão, levando muitas vezes ao abandono do tratamento11, sendo desta forma um dos maiores desafios tanto para o paciente como para os profissionais colaboradores do tratamento.

Uma vez que vários fatores estão envolvidos na adesão ao tratamento, alguns instrumentos têm sido desenvolvidos e validados para que possam abranger o caráter multidimensional da adesão, focando tanto nos aspectos relacionados aos medicamentos como os comportamentais12. Vários métodos têm sido propostos na tentativa de aferir a adesão em doenças crônicas (métodos subjetivos, baseados na autorrelato do paciente ou do profissional de saúde; métodos objetivos, que incluem a contagem de comprimidos, os registros da dispensação de medicamentos e os sistemas eletrônicos; métodos fisiológicos que consideraram a monitorização sérica e os marcadores biológicos como LT CD4 e carga viral), contudo nenhum apresenta acurácia perfeita para definir a situação real de uso dos medicamentos pelo paciente. Considerando esse cenário, o farmacêutico pode auxiliar o usuário, criando um vínculo em relação a profissional-usuário, onde durante 0 acolhimento, são informadas condições para minimizar e evitar efeitos colaterais a fim de melhorar a sua qualidade de vida13. A dispensação da TARV na farmácia é um método facilmente executado pelos farmacêuticos, sendo o registro da dispensação de medicamentos bastante útil para monitorizar a adesão para um número elevado de doentes e quando o doente apenas recebe medicamentos num único local e para intervalos de tempo regulares 14

O Sistema de Controle Logístico de Medicamentos Antirretrovirais (SICLOM) é uma ferramenta eletrônica que dá suporte a acões qualificadas da assistência farmacêutica, garantindo a padronização das ações de controle dos medicamentos ARV. Esta ferramenta está disponível na UDM estudada, que se encontra inserida em um hospital público o qual é referência para a região central do Rio Grande do Sul no tratamento do HIV/Aids, onde os pacientes em TARV retiram mensalmente os medicamentos. Desta forma o presente estudo teve como objetivo verificar o perfil sócio demográfico e adesão ao tratamento dos PVHA que iniciaram TARV junto a UDM no ano de 2015.

Metodologia

O estudo transversal, quantitativo, descritivo, retrospectivo, abrangeu 190 pacientes soropositivos para o HIV que

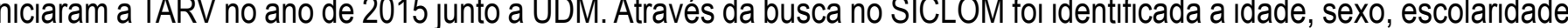
o tempo de tratamento.

Para o estudo da adesão foram excluídos 12 pacientes (8/190) por terem ido a óbito por motivos não conhecido e (4/190) terem sido transferidos para outra UDM. Desta forma, inicialmente foram acompanhados 178 pacientes, verificando-se a frequência na retirada dos medicamentos, para avaliar o grau de adesão a TARV. Pacientes que deixaram de retirar 0 medicamento por mais de 90 dias, foram considerados em abandono de tratamento.

Aliteratura considera com adesão estrita os pacientes que retiram o medicamento em todos os meses do ano, com tomada de $95 \%$ ou mais dos medicamentos dispensados 9 . Uma vez que a verificação da retirada do medicamento não garante que o indivíduo realmente esteja ingerindo a medicação, foram considerados com alta /estrita adesão pacientes que retiraram a TARV durante todos os meses do tratamento, ou que tenham falhado apenas um mês (frequência $\geq 90 \%$ ). Os que não compareceram para a retirada em dois ou três meses foram considerados com adesão regular /adequada (frequência entre 70 e 89\%). Por fim, os pacientes que deixaram de retirar o medicamento mais de quatro meses, alternadamente ou não, foram considerados com adesão insuficiente /baixa ao tratamento (frequência $<70 \%$ ). Esta nomenclatura (Alta/ estrita, adequada/regular e baixa/insuficiente), foi baseada em artigo de Remor, Milner-Moskovics\&Preussler (2007)12. Os dados foram analisados através de estatística descritiva (frequência, porcentagem, média). 0 estudo foi aprovado pelo Comitê de Ética de Pesquisa CAAE 59443616.5.0000.5346, seguindo a Resolução 466/12 do Conselho Nacional de Saúde para a realização de pesquisa envolvendo seres humanos.

Resultados

Na UDM estudada, durante o ano de 2015, 190 pacientes iniciaram a TARV pela primeira vez. Analisando o perfl destes indivíduos, a frequência das faixas etárias encontrada foi variável. Entre os pacientes com menos de 18 anos de idade no ano de 2015, apenas dois eram crianças com menos de um ano, que iniciaram o tratamento devido ter ocorrido transmissão vertical $(1,05 \%)$ e dois eram adolescentes com 16 anos $(1,05 \%)$. Entre os indivíduos com idade a partir de 18 anos, $13,15 \%$ encontravam-se na faixa etária entre 18 a 24 anos; $50,5 \%$, na faixa etária entre 25 a 39 anos; $31,05 \%$; entre 40 a 60 anos e $3,15 \%$ com idade entre 61 a 80 anos.

Verificou-se que a razão entre os sexos foi de 1,18 homens para 1 mulher,sendo 103 indivíduos do sexo masculino $(53,68 \%) 87$ do sexo feminino $(45,15 \%)$. Entre as mulheres, $12(6,21 \%)$ iniciaram o tratamento por serem gestantes com idade média de 29 anos (mínima de19; máxima de 40 anos). A cor/raça predominante foi a branca $(61,05 \%)$ seguido 
da cor parda e negra. Excluindo-se as duas crianças, quanto ao estado civil, houve predomínio de indivíduos solteiros $(54,73 \%)$. Em relação ao grau de escolaridade $22 \%$ relataram menos de 7 anos, e $58,4 \%$ mais de 8 anos de escolaridade. A escolaridade não foi informada por $19,47 \%$ dos pacientes. (Tabela 1).

Tabela 1: Perfil dos pacientes que iniciaram a terapia antirretroviral em 2015. (UDM/Região central-RS).

\begin{tabular}{|lrr|}
\hline Característica & $\mathrm{N}$ & \multicolumn{1}{c|}{$\%$} \\
\hline Corl Raça & & \\
Branca & 116 & 61,05 \\
Parda & 36 & 18,94 \\
Preta & 32 & 16,84 \\
Não informada & 6 & 3,17 \\
Total & 190 & 100 \\
\hline & & \\
\hline Estado civil & & \\
Solteiro & 102 & 54,3 \\
Casado & 46 & 24,5 \\
Divorciado & 19 & 10,1 \\
Viúvo & 6 & 3,2 \\
Não informado & 15 & 7,9 \\
Total & 188 & 100 \\
& & \\
\hline Escolaridade (anos) & & \\
1 a 3 & 5 & 2,7 \\
4 a 7 & 35 & 18,6 \\
8 a 11 & 56 & 29,8 \\
Mais de 12 & 55 & 29,3 \\
Não informado & 37 & 19,6 \\
Total & 188 & 100 \\
\hline
\end{tabular}

*excluído as duas crianças com menos de um ano de idade.

Entre estes pacientes atendidos, a grande maioria faz o acompanhamento clínico através do serviço público (84,21\%) sendo que desses $47,89 \%$ recebem atendimento pelo CTA do município, $50 \%$ são atendidos pelo serviço de infectologia do hospital onde a UDM encontra-se inserida, e 2,10\% não informa o local de atendimento médico. Em $4,21 \%$ dos pacientes, 0 tratamento foi iniciado de acordo com o atual protocolo clinico e diretrizes de tratamento do Ministério da Saúde, onde o tratamento é oferecido para o indivíduo HIV positivo, independente da sua situação clínica8.

Entre os 178 pacientes inicialmente acompanhados, 22 abandonaram o tratamento (11,83\%), todos com idade superior a 20 anos, faixa etária predominante na amostra estudada. Entre os pacientes que abandonaram o tratamento, estavam duas gestantes, que apresentaram carga viral inferior a 50 cópias $/ \mathrm{mL}$ na última avaliação solicitada pelo clínico responsável.

Desta forma, foi possivel acompanhar a adesão a TARV por pelo menos seis meses de 156/190 pacientes atendidos na UDM no ano de 2015. Amaioria destes pacientes apresentou adesão satisfatória a TARV. Entre os pacientes acompanhados, 138/156 apresentaram alta adesão, 6/156 apresentaram adesão regular, 12/156 adesão insuficiente (Tabela 2).
Tabela 2: Frequência de adesão à terapia antirretroviral de pacientes que mantiveram o tratamento iniciado em 2015 (UDM/região central-RS).

\begin{tabular}{|l|c|}
\hline Grau de Adesão & Frequencia (\%) \\
Alta adesão & 88,5 \\
Adesão regular & 3,84 \\
Adesão insuficiente & 7,7 \\
Total & 100 \\
\hline
\end{tabular}

Considerando-se apenas as gestantes em uso de TARV acompanhadas no período de estudo $(n=10), 90 \%$ apresentaram alta adesão ao tratamento, e em 10\% a adesão foi insuficiente no inicio da gestacão, passando esta paciente a tomar a medicação corretamente no final da gravidez, conseguindo manter a carga viral inferior a 50 cópias/ $\mathrm{mL}$ no momento do parto.

Discussão

A rápida disseminação da infecção pelo HIV é evidenciada pelo fato da doença vir acometendo indivíduos de diferentes culturas e espaços, assim como atingindo indivíduos de muitas faixas etárias 15,16 . Vale ressaltar que antes da portaria MS N01.271, de 6 junho 2014, os casos notificados no Brasil, referiam-se apenas ao número de indivíduos que desenvolveram aids e não ao número de indivíduos soropositivos para o HIV uma vez que estes não eram considerados casos de notificação compulsória. Desta forma, o Brasil tem registrado, anualmente, uma media de 41,1 mil casos de aids nos últimos cinco anos 2. Em dezembro de 2013, o Brasil tornou-se o primeiro país em desenvolvimento e o terceiro do mundo a recomendar, através do Protocolo e diretrizes para tratamento do HIV8, o início imediato da TARV para todas as PVHA, independentemente da contagem de LT CD4+. Deve-se então considerar que o perfil de indivíduos que iniciaram a TARV em 2015, representado no grupo estudado, por crianças, adolescentes, e indivíduos adultos em diferentes faixas etárias e graus de escolaridade, refletem o caráter heterogêneo da epidemia e as mudanças que ocorreram no enfrentamento da mesma. A faixa etária predominante entre os indivíduos que iniciaram a retirada de medicamentos para a TARV (2015), independente de estarem desenvolvendo a aids, foi de 25 a 39 anos (50\%), semelhante a mesma faixa etária predominante, em ambos os sexos, entre os casos de aids notificados em 2006 e 2015 no Brasil2.

Embora a razão entre os sexos tenha diminuindo no decorrer dos anos, o Brasil apresenta uma maior prevalência de aids entre os pacientes do sexo masculino/feminino (razão 2,4)2, Considerando-se que a razão de sexos apresenta diferenças regionais importantes, com a região Sul apresentando uma participação maior das mulheres nos casos de aids notificados (razão de sexos de 17 homens para cada 10 mulheres)2, não surpreende a razão entre sexo de 12 homens/10 mulheres entre os pacientes que iniciaram a TARV na UDM estudada, o que reflete uma epidemia distribuída de forma quase igualitária, lembrando que neste período já estava sendo indicada a TARV para indivíduos que eram portadores de HIV, sem presença de aids.

No que se refere a gestantes, deste o ano 2000, é obrigatória a notificação da gestante portadora do HIV (Portaria no 993/GM - 04/09/00). Em 2006 a taxa observada no Brasil foi de 2,1 casos/mil nascidos vivos, apresentando um aumento de $28,6 \%$ em relação ao ano de 2016, com taxa descrita de 2,7 casos/mil nascidos vivos2. Este crescimento está vinculado a implementação de testes rápidos para o diagnóstico do HIV17, que tem feito com que a taxa de detecção em gestantes nos últimos dez anos apresente tendência de aumento.

Entre os indivíduos adultos que iniciaram a TARV no presente estudo, encontravam-se 12 gestantes, demonstrando que o fato de conhecer a situação sorológica positiva para HIV não impede uma gravidez, por vezes não planejada. E ainda, possivelmente devido ao conhecimento de que a instituição de TARV precoce durante a gravidez diminul a probabilidade de transmissão vertical da infeç̧ão, o desejo de ser mãe não é suplantado pelo diagnóstico, fato já abordado por outros autores 18.

Porém, a conscientização e o nível desejado de adesão a TARV para evitar a TV nem sempre são atingidos. Entre os indivíduos que iniciaram a TARV em 2015, 10\% das gestantes não aderiram de forma total a TARV e 1,05\% eram crianças infectadas pelo HIV em decorrência da TV. Uma adesão adequada a TARV é crucial para que diminuam os riscos de TV intra-útero e durante o parto, bem como os riscos do desenvolvimento da aids. Desta forma, devido às dificuldades encontradas no início do tratamento, a atenção do profissional do serviço para com o paciente, deve ser Saúde (Santa Maria), Vol. 43, n. 3, p. 1-8, maio./ago, 2017 
redobrada, reforçando a conscientização e comprometimento com a terapia medicamentosa, uma vez que a não-adesão é considerada como um dos maiores problemas para a efetividade do tratamento19.

Para avaliar a adesão ao tratamento, entre os diversos métodos empregados, o monitoramento eletrônico de medicamentos, contagem de comprimidos e registros de retiradas de medicamentos no sistema de dispensação da UDM, encontram-se como os mais fáceis de serem realizados em uma UDM20. Uma vez que a TARV é distribuída mensalmente, a data da retirada no mês corrente pode ser comparada com a data espera para o regresso do paciente, tendo-se assim uma coerência nos dias da dispensação dos medicamentos. Já foi relatado que pacientes que retiram medicamento na data certa tendem a toma-los mais corretamente do que aqueles que atrasam os dias da retirada21.

Devido a grande importância desta questão, diversos estudos focando a adesão a TARV têm sido desenvolvidos ao longo dos anos. Estudo de revisão de Garbin et al (2017), avaliando artigos publicados entre os anos de 2009 a 2015 que estudaram a adesão a TARV, mostrou que a taxa de não adesão variou de $18 \%$ à $74,3 \%$ nos artigos analisados . Estas taxas podem ter apresentado uma variação tão ampla devido a variantes presentes nos diferentes estudos, como a complexidade do serviço onde o paciente é atendido. Nemes (2009) verificou que grupos de pacientes tratados em serviços com número menor que 100 pacientes apresentaram risco de não-adesão maior que os serviços com mais de 500 pacientes23. Desta forma, o alto grau de adesão encontrada entre a maior parte dos indivíduos avaliados no presente estudo $(88,5 \%)$ está de acordo com a estrutura do hospital público onde está localizada a UDM, que considerado um hospital de grande porte, alta complexidade e referência regional no tratamento ao HIV, com excelência no serviço prestado, e que atende mais de 1000 pacientes HIV/mês. Porém, mesmo com todo esforço realizado pelos profissionais de saúde envolvidos, o risco de abandono do tratamento durante a TARV continua sendo uma realidade. Em 2012 foi realizado um estudo de adesão na UDM em estudo, onde se verificou uma taxa de abandono de 15,2\% entre os indivíduos avaliados, considerando a frequência na retirada de medicamentos e os resultados de CV, que um critério definitivo para avaliar a adesão24. Nota-se que a taxa de abandono melhorou desde então, uma vez que no presente estudo, no ano de 2015 apenas $11,83 \%$ dos indivíduos abandonaram o tratamento, mostrando uma leve queda quando comparada a taxa encontrada no ano de 2012 na mesma instituição. Este desafio também é enfrentado em outros serviços de saúde. Em Salvador na Bahia, usuários de TARV foram acompanhados durante seis meses e aproximadamente $15 \%$ dos pacientes encontrou-se em situação de abandono de tratamento, relatando um índice de $25 \%$ de adesão insatisfatória e insuficiente 19 .

Felizmente, apesar de todas as dificuldades enfrentadas, o tratamento brasileiro tem reconhecimento internacional, sendo considerado como mais eficiente que a média global. Segundo relatado por agencias de noticias, as mortes em decorrência do vírus HIV no País caíram a uma taxa anual de 2,3\% entre 2000 e 2013, enquanto a média global apresentou uma queda de $1,5 \%$ ao ano25.

Para que estes índices melhorem, é necessário que cada vez mais se invista na qualidade da relação com a equipe de saúde, na compreensão da necessidade do tratamento pelo paciente, principalmente nos primeiros meses após 0 início da terapia, quando é comum a manifestação de reações adversas, erros no uso dos antirretrovirais, esquecimentos de dose e outros fatores que afetam negativamente a adesão. Desta forma, apesar de ainda não ter sido atingido um grau de excelência, no que se refere à adesão a TARV, é possivel considerar que a atenção farmacêutica no momento da dispensação do medicamento, junto com todo o suporte oferecido por uma equipe multiprofissional composta po médicos, enfermeiros, psicólogos, assistente social, que atendem os indivíduos portadores de HIV, tem realizado em trabalho eficiente, refletido no bom índice de adesão encontrado no presente estudo.

\section{REFERÊNCIAS BIBLIOGRÁFICAS}

1. Loreto $\mathrm{S}$, Azevedo-Pereira JM. A infecção por HIV-importância das fases iniciais e do diagnóstico precoce. Acta Farmacêutica Portuguesa. 2012;1(2): 5-17.

2. BRASIL. Ministério da Saúde. Programa Nacional de DST e AIDS. Boletim epidemiológico HIV. Brasília, DF, Departamento de IST, AIDS e Hepatites Virais 2016. [Citado em 8 de março de 2017].Disponível em: http://www.aids.gov.br/sites/default/files/anexos/publicacao/2016/59291/boletim_2016_1_pdf_16375. pdf.

3. Villarinho MV, Padilha MI, Berardinelli LMM, Borenstein MS, Meirelles B HS, Andrade SR. Políticas públicas de saúde face à epidemia da AIDS e a assistência às pessoas com a doença. Rev Bras Enferm. 2013;66 (2): 271-277.

4. Silva ACO, Reis RK, Nogueira JA, Gir E. Quality of life, clinical characteristics and treatment adherence of people living with HIV/AIDS. Rev. Latino-Am. Enfermagem. 2014; 22(6): 994-1000.

5. Oliveira E A, Labra ME, Bermudez, J. A produção pública de medicamentos no Brasil. Cadernos de Saúde Pública. 2006; 22 (11): 2379-2389.

6. Montaner JS, Lima VD, Harrigan PR, Lourenço, L, Yip, B, Nosyk, B et al Expansion of HAART coverage is associated with sustained decreases in HIV/AIDS morbidity, mortality and HIV transmission: the "HIV Treatment as Prevention" experience in a Canadian setting. PLoS One, [supl.]. 2014; 9 (2):87872.

7. Cohen MS, Chen YQ, McCauley M, Gamble T, Hosseinipour MC, Kumarasamy N, Hakim JG et al. Prevention of HIV-1 Infection with Early Antiretroviral Therapy. N. Engl. J. Med. [supl]. 2011; 365:493-505.

8. BRASIL Ministério da Saúde. Protocolo clínico e diretrizes terapêuticas para manejo da infecção pelo HIV em adultos. Brasilia DF: Departamento de DST, Aids e hepatites virais; 2015. [Citado em 7 mai 2016]. Disponível em:http://www.aids.gov.br/sites/default/files/anexos/publicacao/2013/55308/ protocolofinal_31_7_2015_pdf_31327.pdf

9. Paterson D, Swindells S, Mohr J, Brester M, Vergis E, Squier C, Wagener MM, Singh N. Adherence to protease inhibitor therapy and outcomes in patients with HIV infection. Annals Intern Med. 2000; 133(1):21

10. Wensing, AM., Calvez, V, Günthard, HF, Johnson, VA, Paredes, R, Pillay, D, et al. Special Contribution 2014 Update of the Drug Resistance Mutations in HIV-1. Topics in antiviral medicine. 2014; 22(3):642-650.

11. De La Torre-Lima J, Aguilar A, Santos J, Jiménez-Oñate F, Marcos M, NúñezV, et al \& Málaga Infectious Disease Group. Durability of the First Antiretroviral Treatment Regimen and Reasons for Change in Patients With HIV Infection. HIV Clinical Trials. 2014; 15(1): 27-35.

12. Remor E, Milner-Moskovics J, Preussler G. Adaptação brasileira do "Cuestionario para La Evaluación de La Adhesión al Tratamiento Antiretroviral". Rev Saúde Pública. 2007; 41(5): 685-694.

13. Polejack L, Seidl EMF Monitoramento e avaliação da adesão ao tratamento antirretroviral para HIV aids: desafios e possibilidades. Ciência \& Saúde Coletiva. 2010,15(Supl. 1): 1201-1208.

14. Paterson DL, Potoski B, Capitano B. Measurement of adherence to antiretroviral medications. Journal of acquired immune deficiency syndromes. 2002; 31: 103-106.

15. Brito AM, CastilhoEA, Szwarcwald CL. AIDS e infecção pelo HIV no Brasil: uma epidemia multifacetada. Rev. Soc. Bras. Med.Trop. 2001; 34: 207-217.

16. Bertoni RF, Bunn K, Silva DJ , Traebert J. Perfil demográfico e socioeconômico dos portadores de HIV/AIDS do Ambulatório de Controle de DST/AIDS de São José, SC. Arquivos Catarinenses de Medicina. 
2010; 39(4): 75-79.

17. BRASIL, Ministério da Saúde, Protocolo clínico e diretrizes terapêuticas para prevenção da transmissão vertical de HIV, síflis e hepatites virais. 2015c. Disponível em: http://www.aids.gov.br/sites/ default/files/anexos/publicacao/2015/58572/pcdt_transmissao_vertical_miolo_10_08_pdf_5557e.pdf, [acessado em: 04 junh 2017].

18. Levandowski DC, Pereira MD, Dores SDS, Ritt GC, Schuck, LM, Sanches IR. Experiência da gravidez em situação de seropositividade para o VIH: Revisão da literatura brasileira. Análise Psicológica. 2014;32(3), 259-277.

19. Silva JAG, Dourado I, Brito AM, Silva CAL. Fatores associados à não adesão aos antirretrovirais em adultos com AIDS nos seis primeiros meses da terapia em Salvador, Bahia, Brasil. Cad. Saúde Pública. 2015; 31(6):1188-119. [Citado em 12 mar 2017]. Disponível em: http://www.scielo.br/pdf/csp/v31n6/0102-311Xcsp-31-6-1188.pdf

20. Polejack L, Seidl EMF. Monitoramento e avaliação da adesão ao tratamento antirretroviral para HIV/ AIDS: desafios e possibilidades.Ciênc Saúde Coletiva. 2010; 15(1): 1201-8.

21. Mcpherson-Baker S, Schneiderman N. A measurement model of medication adherence to highly active antiretroviral therapy and its relation to viral load in HIV-positive adults. Aids Patient Care and STDs. 2006; 20(10): 701-711.

22. Garbin CAS, Gatto RCJ, Garbin ARI. Adesão à terapia antirretroviral em pacientes HIV soropositivos no Brasil: uma revisão da literatura. Arch Health Invest . 2017; 6(2): 65-70.

23. Nemes MIB. Adesão ao tratamento, acesso e qualidade da assistência em Aids no Brasil. Rev. Assoc. Med. Bras. 2009; 55(2): 207-212.

24. Vielmo L, Campos MMA, Beck ST, Andrade CS. Atenção farmacêutica na fase inicial de tratamento da AIDS como fator importante na adesão aos antirretrovirais. Rev. Bras. Farm. 2014; 95(2): 617-635

25. Dia Mundial de Luta Contra a Aids: conquistas e desafios. RADIS Fundação Oswaldo Cruz, Programa Radis de Comunicação e Saúde, da Escola Nacional de Saúde Pública Sergio Arouca (n148), 5,Disponível em http://www6.ensp.fiocruz.br/radis/sites/default/files/radis_148_web.pdf, acessado em maio 2017. 